\title{
Oral Manifestations of Inflammatory Bowel Diseases: Two Case Reports
}

\author{
Manoela Seadi Pereira and Maria Cristina Munerato
}

\begin{abstract}
Inflammatory bowel diseases (IBD) are known as chronic inflammatory disorders of the digestive tract, represented mainly by Crohn's disease (CD) and ulcerative colitis (UC). Among the main oral manifestations of IBD are cobblestoning of the oral mucosa, labial swellings with vertical fissures, pyostomatitis vegetans, angular cheilitis, perioral erythema, and glossitis. In this sense, understanding these nosological entities by dentists would help reach early and differential diagnosis. Thus, two case reports are presented and discussed based on theoretical references obtained by a literature review. The first case report refers to an adult patient whose IBD diagnosis was established after stomatological assessment. The second case was a patient with $C D$ diagnosed in childhood with characteristic oral lesions.
\end{abstract}

Keywords: Aphthous stomatitis; Crohn's disease; Inflammatory bowel diseases; Oral lesions; Oral manifestations; Ulcerative colitis

$\mathrm{I}$ nflammatory bowel diseases (IBD) are characterized as a chronic inflammatory process in the digestive tract wall caused by the interaction between microorganisms and a genetically susceptible host. Its multifactorial etiology has not been clarified, though risk factors for IBD include smoking habit, diet, vitamin D deficiency, drug abuse, geographic and epidemiologic factors, and social and psychological stress. ${ }^{1-6}$ Crohn's disease (CD) and ulcerative colitis (UC) are among the main forms of IBD. It is known that CD may affect any region of the gastrointestinal system. As a rule, the prevalence of IBD is high in industrialized countries like Canada and northern Europe, though recent studies have revealed that prevalence and incidence of these diseases are increasing in eastern nations, possibly due to generalized behavioral and environmental changes. In addition, while in the West CD is slightly more prevalent in women, in the East men are more often diagnosed with the disease. .,3, $, 7,-9$ In turn, UC is an immune condition that results from an abnormal interaction between the colon microbiota and the intestinal mucosa immune cells in a genetically susceptible individual. Equally prevalent in both genders, UC is slightly more prevalent in men in the West. ${ }^{10}$
Oral manifestations of $\mathrm{CD}$ are observed in $8 \%$ to $10 \%$ of patients and may be considered specific or unspecific. ${ }^{11-13}$ Specific manifestations are comparatively rare and sometimes precede gastrointestinal lesions, which may evolve with time. These manifestations include cobblestoned oral mucosa, granular gingival swelling of hyperplastic aspect, labial swelling accompanied by vertical fissures, and deep and linear ulcers associated with hyperplastic mucosa folds on the vestibule fold. Though indicative of $\mathrm{CD}$, such conditions are not correlated with the dynamics of the disease. ${ }^{14,15}$ Nonspecific oral lesions include recurrent aphthous stomatitis (RAS), pyostomatitis vegetans, angular cheilitis, persistent submandibular lymphadenopathy, decreased saliva production, halitosis, caries, gingivitis, candidiasis, odynophagia, dysphagia, increased intrinsic salivary glands, perioral erythema, recurrent oral abscesses, glossitis, pale mucosa, lichen planus, and metallic dysgeusia. ${ }^{14,16}$

Oral manifestations of UC may emerge as aphthous ulcers or superficial hemorrhagic ulcers and angular cheilitis. As a rule, extraintestinal manifestations parallel active oral involvement. ${ }^{11,17}$ Oral manifestations are less frequent, though they are similar to the unspecific expression of $\mathrm{CD} .^{14,18}$
Corresponding Author: Dr. Maria Cristina Munerato, Faculdade de Odontologia UFRGS, Rua Ramiro Barcelos 2492, Departamento de Odontologia Conservadora (DOC), Porto Alegre, RS. Brazil. 90035-003, Tel: +55 5I 33598248,

Fax: +55 5 I 33085005, Email: mcmunerato@gmail.com
Received: September 28, 2015

Revised: November 17, 2015

Accepted: December 14, 2015

doi: $10.3121 / \mathrm{cmr} .2015 .1307$ 
Pyostomatitis vegetans is the only condition that is more prevalent in UC patients, compared to individuals with CD. ${ }^{11,12,14,19,20}$

Due to the high prevalence of oral UC, knowledge about these conditions is a useful tool in differential diagnosis, since an improvement in the clinical picture concerning the primary disease will influence the recovery of oral health. Moreover, considering that oral lesions may precede gastrointestinal signs and symptoms, the dentist may play an important role in the early diagnosis of IBD. In this scenario, we describe two cases of IBD with associated oral manifestations.

\section{Case Presentation I}

A 32-year-old Caucasian male was referred to the Stomatology Unit of the Hospital de Clínicas de Porto Alegre (HCPA), Federal University of Rio Grande do Sul (UFRGS), Porto Alegre, Brazil, to treat recurrent ulcers on the oral mucosa. The patient's clinical history dates back to 1994, when he left his hometown in the state of Rio Grande do Sul, Brazil for treatment of a condition suspected to be conjunctivitis in both eyes. At that time, clinical evaluation revealed not only the ocular lesion but also lesions on the mouth compatible with larger RAS, with suspicion of Stevens-Johnson syndrome. However, the patient could not remember having received any specific treatment based on that diagnosis at that time.
In 2007 the patient was referred to the Stomatology Unit of HCPA, presenting with large and persistent oral ulcers. Biopsy of one of the ulcers and a therapeutic investigation failed to lead to an accurate diagnosis. As with the other institutions in which the patient had sought treatment, no bond was formed with the medical team, since he missed several appointments. This loss of continuity of the investigation process may have delayed the diagnosis of the primary disease.

Five years later, the patient returned to the Stomatology Unit of HCPA complaining of bilateral RAS on the jugal mucosa. The lesions exhibited white or yellowish pseudomembrane with an erythematous halo. The lesions were intensely painful and, according to the patient, never subsided totally, with numerous new ulcers emerging frequently. In addition, both upper and lower lip mucosa and the bilateral jugal mucosa were rather fibrous in aspect, with scars that indicated previous large RAS (figures 1, 2A \& B, 3A \& B). The patient submitted to another biopsy of the ulcers on the oral mucosa. Anatomical and pathological diagnosis established chronic inflammation on the squamous mucosa accompanied by acanthosis and parakeratosis.

Oral lesions were controlled with dexamethasone $0.05 \%$, and the stomatology team proceeded with the investigation into

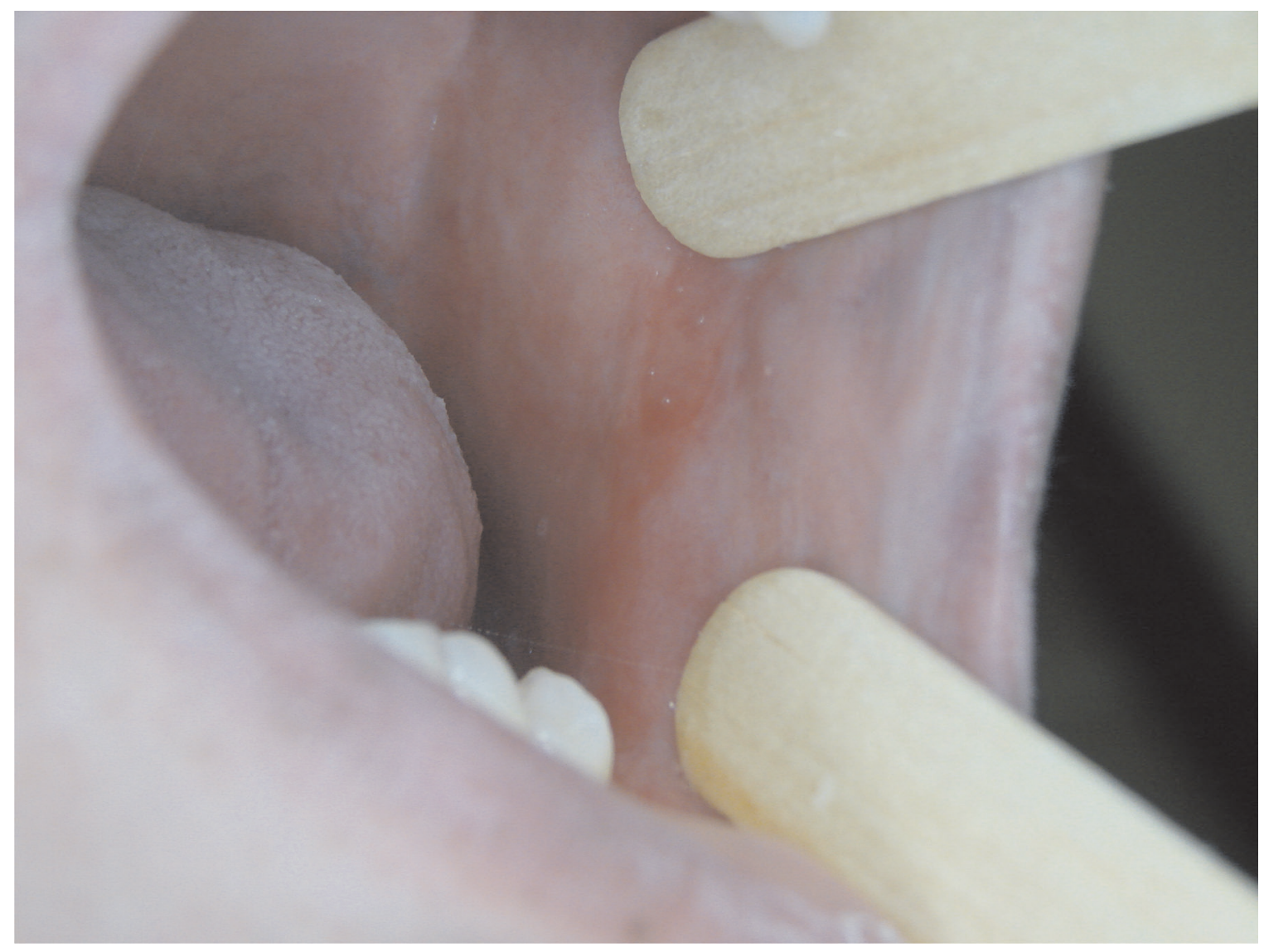

Figure 1. Left jugal mucosa with scars, erythematous areas, and loss of tissue elasticity. 


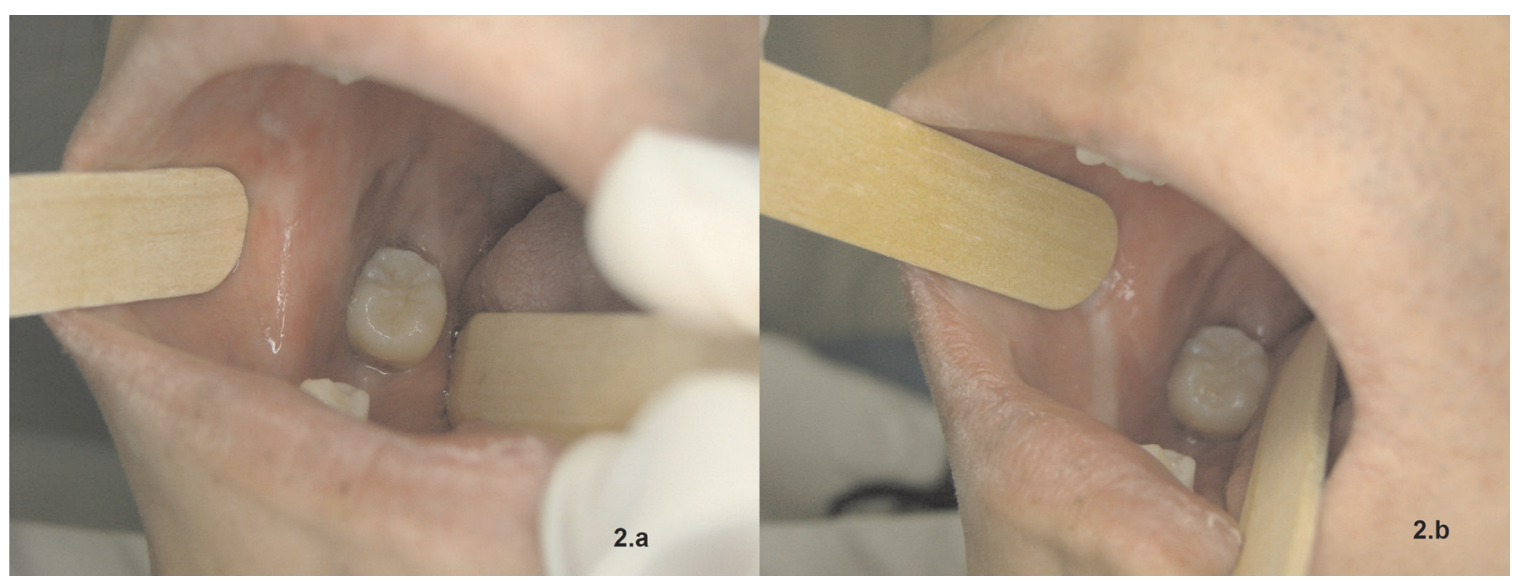

Figure 2. (A) Scars on the right jugal mucosa and relevant painful ulceration near the lower right molar. (B) Scar fibrosis with ischemia of mucosa upon distention, making inspection of oral cavity more difficult.

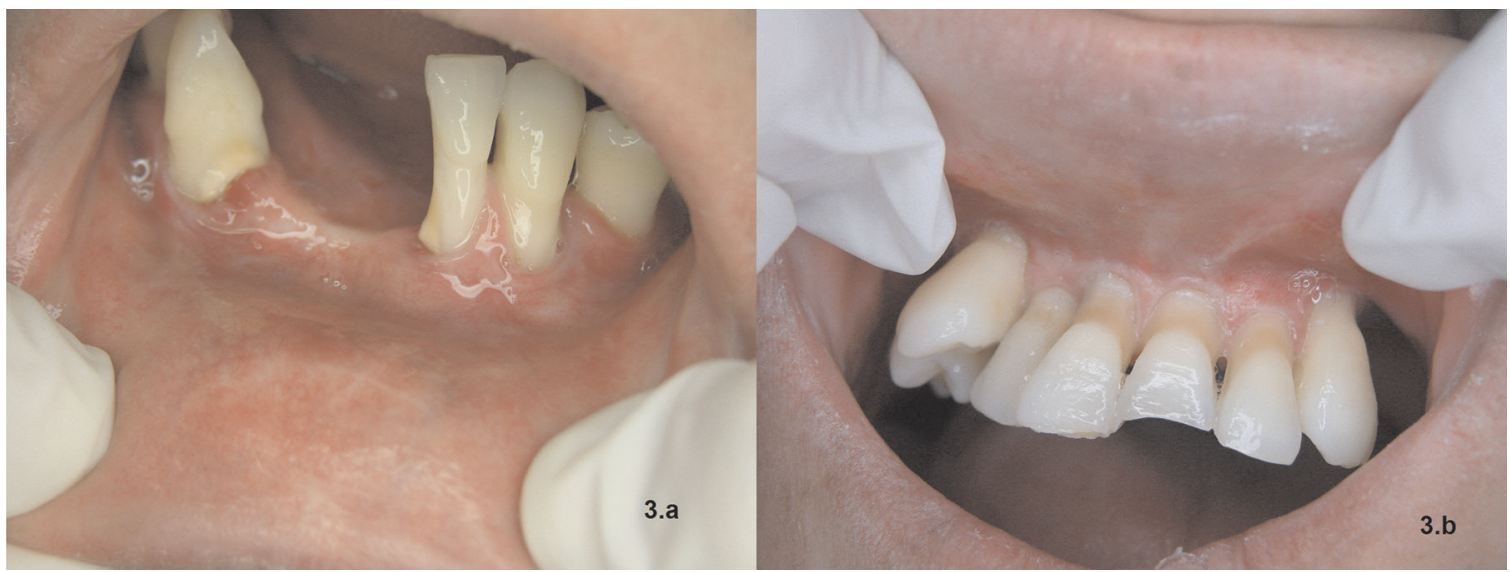

Figure 3. (A) Gingival recession on the anterior lower teeth, with changed vestibule fold and scars on the lower labial mucosa. (B) Gingival recession on the anterior upper teeth, gingiva growing towards the vestibule fold, and presence of important scar fibrosis.

the reason why the RAS was so severe. The patient answered some specific questions about autoimmune diseases, reporting intense and frequent diarrhea episodes since childhood, which coincided with the emergence of recurrent oral ulcers. The medical team then decided to carry out a differential diagnosis of IBD with oral extraintestinal manifestations. Since the patient had RAS and presented several ocular lesions that lead to progressive blindness starting at the age of 13, the hypotheses of $\mathrm{CD}$ and $\mathrm{UC}$ were considered.

The patient was then referred to the gastroenterology service of HCPA for the investigation of the gastrointestinal condition. Biopsy of specimens of the intestinal mucosa collected from the transverse colon, left colon, rectum, and rectosigmoid junction indicated chronic inflammation with architectural distortion, irregular surface, increased and redistributed inflammatory infiltrate in lamina propria in the colon and rectal mucosae, in addition to cryptitis and crypt abscesses. Examination of the clinical record and the biopsy of intestinal wall specimens indicated UC as a systemic condition.

The patient's diarrhea episodes were then treated with mesalazine, with good results. RAS was treated initially with dexamethasone $0.05 \%$ mouthwash once a day for 3 months. At a follow-up appointment, the patient reported total subsidence of the lesions. The patient was instructed to use the dexamethasone $0.05 \%$ mouthwash whenever he noticed any recurrence of symptoms. Currently, he is under transdisciplinary medical follow-up, and his primary disease is under control. The therapeutic response obtained with mesalazine lends strength to the diagnosis of UC.

\section{Case Presentation 2}

A 17-year-old Caucasian male was diagnosed with $\mathrm{CD}$ in 2006, at the age of 8 years. The patient had started treatment with the gastroenterology service of HCPA in February 2011, having been referred by another health unit that was not appropriately equipped to treat cases of greater complexity. Within a 6-year period, diagnosed co-morbidities included iron-deficiency anemia, perianal fistulae with purulent secretion (a manifestation of $\mathrm{CD}$ ), left inguinal hernia, cholestatic hepatitis caused by herpes zoster, depression, and a series of opportunistic infections that started with treatment.

The first appointment at the Stomatology Service of HCPA was in January 2012, when the patient presented with 
erythematous fissures on lip commissures, compatible with angular cheilitis (figure 4), and bilateral ulcers on the vestibule fold, consistent with RAS. Coalescing hyperplastic cobblestone lesions typical of $\mathrm{CD}$ were also observed bilaterally on the jugal mucosae (figures $5 \mathrm{~A}$ and $\mathrm{B}$ ).

Patient 2 had been taking prednisone $20 \mathrm{mg}$ as prescribed by the Gastroenterology team of HPCA as of the first appointment with the stomatology team, in January 2012. Since this medical drug is used in the treatment of autoimmune oral ulcers, prescription of an additional medicament was ruled out. However, the dose of this systemic corticosteroid was gradually cut down to $5 \mathrm{mg}$ as a maintenance dose, according to the evolution of CD (in December 2013). The decision made by the gastroenterology team to adjust the prednisone regimen by changing dose or even discontinuation in order to treat $\mathrm{CD}$ more effectively prompted the stomatology team to prescribe an aqueous solution of clobetasol propionate $0.05 \%$ in combination with nystatin $100,000 \mathrm{IU} / \mathrm{mL}$ as mouthwash (three times a day, for seven days), also with good results to treat RAS (Fig 5C and D). Angular cheilitis was treated with miconazole $2 \%$ oral gel and nystatin $100,000 \mathrm{IU} / \mathrm{mL}$ mouthwash four times a day for 15 days, with significant improvement.

Currently CD is partially controlled with injections of adalimumab (40 mg). The patient is under regular follow up with the gastroenterology team, and has been instructed to return to the Stomatology Unit of HCPA if the prescribed mouthwash is ineffective to control oral ulcers.

\section{Discussion}

Prevalence of $\mathrm{CD}$ has been estimated at 30 to 50 cases in 100,000 inhabitants in western countries, though it is considerably higher in the East. While incidence of CD is slightly higher in women than in men in western nations, in the East the prevalence is higher in men. As for CU, prevalence is not considered to vary between sexes, though studies have pointed to slightly higher values in men. ${ }^{8}$ Smoking may be a risk factor for $\mathrm{CD}$, differently from $\mathrm{UC} .^{2,3,5,7-9}$ In both cases reported here, the patients were males who had never smoked.

A study of 1,649 individuals with IBD determined the prevalence, incidence, and risk factors for extraintestinal manifestations of the disease. ${ }^{21}$ Articular manifestations, such as arthritis, and oral findings like aphthoid ulcers were the most common extraintestinal manifestations observed prior to the diagnosis of IBD, affecting $26 \%$ and $21 \%$ of the population investigated, respectively. ${ }^{21}$

Oral manifestations of IBD were first described in patients with CD. In most cases, intestinal involvement precedes oral manifestations, though it is estimated that between $5 \%$ and $10 \%$ of patients exhibit oral manifestations primarily. ${ }^{14,21}$ Oral

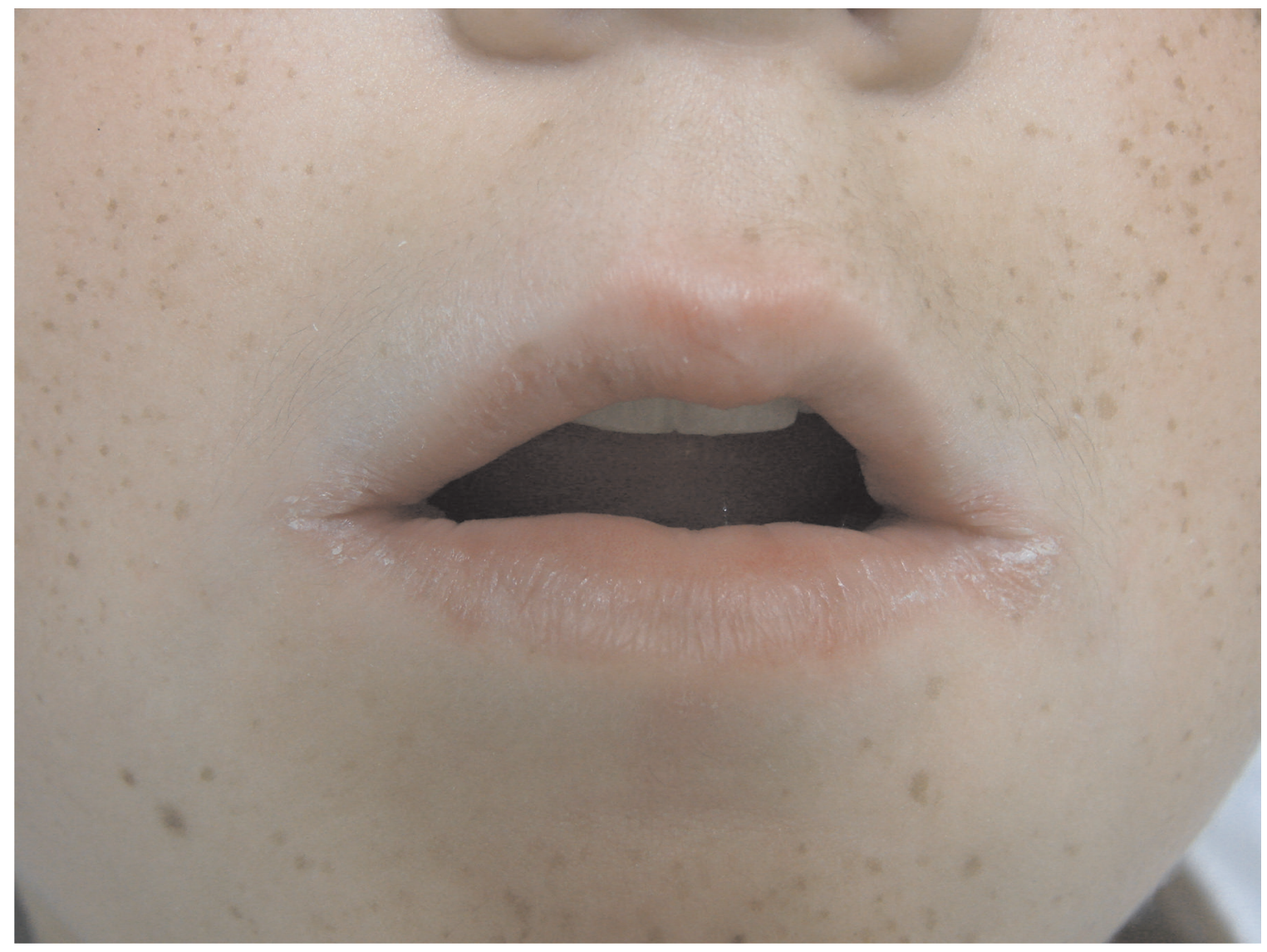

Figure 4. Erythematous fissures on lip commissures and skin desquamation compatible with angular cheilitis. 


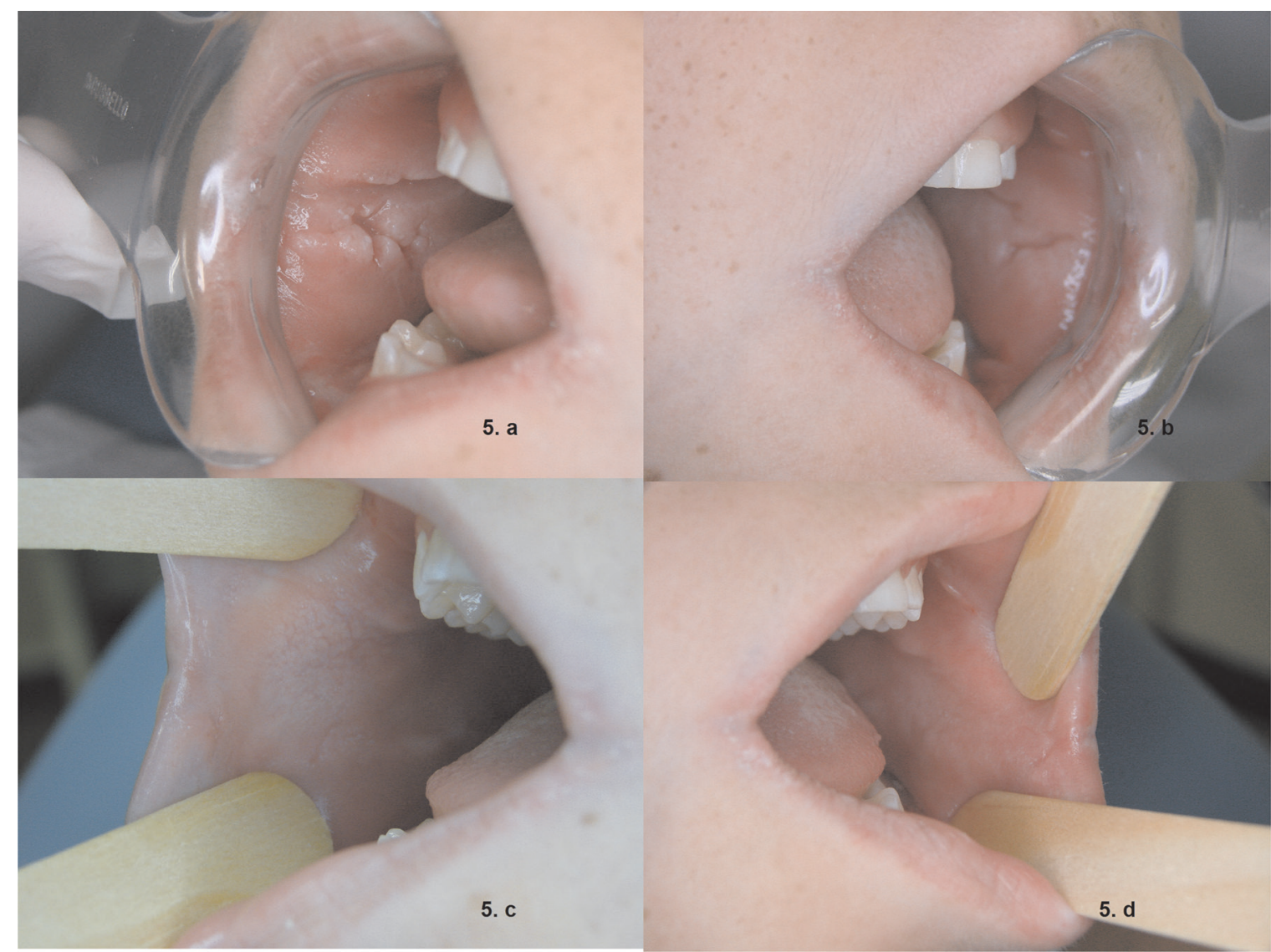

Figure 5. Bilaterally cobblestoned jugal mucosa (A and $B)$, and subsidence after partial treatment of $C D(C$ and $D)$.

lesions are more common in $\mathrm{CD}$ than in $\mathrm{UC}$ and in men and children, ${ }^{14,15}$ as observed in our second patient, a male who had developed extraintestinal manifestations already in childhood.

The patients described in both our cases presented with mucocutaneous and oral manifestations. Patient 1 also exhibited ocular manifestations, which occur in approximately $10 \%$ to $12 \%$ of individuals with IBD. ${ }^{17,22}$ In this sense, it is critically important to monitor ocular symptoms in potential IBD cases, since severe episcleritis, uveitis, scleritis, and cornea diseases may resemble conjunctivitis, with risk of blindness if the appropriate treatment is not prescribed. ${ }^{21,22}$ This is exactly what happened with patient 1 , since the early ocular manifestation of IBD mistakenly led to the diagnosis of conjunctivitis, which, after a series of complications, resulted in blindness.

Deficiencies in diet and in the absorption of essential vitamins and nutrients like zinc, for example, may also trigger mucocutaneous manifestations such as periorofacial dermatitis including erythematous, dry, and desquamating plaques that may evolve into erosive or vesicular and blister-like lesions, apart from glossitis. Iron deficiency leads to angular stomatitis and painful depapillation of the tongue. ${ }^{11,23}$ In patient 2, who was diagnosed with $\mathrm{CD}$, the constant vitamin and nutritional deficiencies, especially in iron and zinc, may have a strong correlation with angular cheilitis and erosive and crusty lesions on the lip commissures and perioral region.

In addition to the mucocutaneous manifestations of IBD, anemia is another disease often observed, with approximately one-third of patients presenting with low hemoglobin levels. Chronic intestinal hemorrhage, which reduces iron levels, causes hypochromic and microcytic anemia, impairs the absorption of vitamin B12, and worsens folate deficiency. ${ }^{22}$ These changes were observed in both patients that we reported here.

Patient 2, treated for $\mathrm{CD}$, presented with three different oral lesions, namely intraoral lesions compatible with aphthous ulcers bilaterally across the vestibule fold, hyperplastic cobblestone lesions bilaterally on the jugal mucosa (which are considered indicative of this CD),${ }^{14}$ and perioral lesions, mainly bilaterally on the lip commissures that were diagnosed as angular cheilitis. This clinical finding highlights the role of nutritional deficiencies in the emergence of oral lesions of this kind. Apart from being a nonspecific manifestation of $\mathrm{CD}$, zinc deficiency has been shown to cause desquamating lesions on the periorofacial region, while iron deficiency induces angular cheilitis. ${ }^{11}$

UC causes pain in the left side of the abdomen, diarrhea, weight loss, and rectal hemorrhage, starting gradually and 
worsening within weeks. ${ }^{2,8}$ The condition is diagnosed based on these symptoms and endoscopy findings, usually confirmed by biopsy. This clinical scenario was confirmed during the interview with patient 1 , who reported severe diarrhea and abdominal pain. In addition, the clinical conduct adopted to treat patient 1 included intestinal biopsy to confirm the suspicion of UC, as described in the literature reviewed here.

Pellicer et $\mathrm{al}^{11}$ reported that oral manifestations in UC may occur as aphthous ulcers, superficial hemorrhagic ulcers, or angular cheilitis, and, as a rule, extraintestinal expression coincides with the period when the disease is active. ${ }^{11}$ The lesions detected in patient 1 were compatible with RAS, in agreement with the main oral manifestations of UC described in the literature. Another important variable was the control of oral lesions, whose success depends on the effectiveness of the treatment of IBD, which was confirmed in the literature findings. ${ }^{11}$

Although pyostomatitis vegetans is a rare ulcerous condition in patients with $\mathrm{UC}$, and even rarer in cases of $\mathrm{CD}$, the condition is one of the specific manifestations of IBD. The correct management of the primary disease is essential as a means to cure oral lesions. ${ }^{13,14,16,18,20}$

As confirmed in patient 2, cobblestone lesions are the main oral manifestation of CD. Such lesions are characterized by thickened, firm, and pinkish areas mostly on the labial and jugal mucosa, which may be multifocal, linear, polypoid, or diffuse. Since cobblestone lesions are usually painless, an association with concomitant ulcers has to be considered when the patient complains of pain. Oral extraintestinal manifestations of CD are more often observed in men, and at times occur prior to the disease itself, though they also are detected in patients with confirmed CD. ${ }^{14,24}$

\section{Conclusion}

The two cases described in this report about IBD with oral extraintestinal manifestations show that the association between IBD and oral mucosa lesions and other co-morbidities such as anemias and vitamin deficiencies pose a challenge in clinical stomatology, requiring constant professional training in light of the numerous circumstances in which oral lesions may indicate important primary diseases yet to be diagnosed. In this sense, the dentist could contribute in the effort to reach an early diagnosis of a large number of conditions. Regarding IBD, like $\mathrm{CD}$ and $\mathrm{UC}$, the transdisciplinary work of the gastroenterologist and the stomatologist enables a comprehensive approach to treatment, while also guaranteeing that the therapeutic management of these diseases is appropriate and efficient.

\section{References}

1. Dam AN, Berg AM, Farraye FA. Environmental influences on the onset and clinical course of Crohn's Disease-part 1: an overview of external risk factors. Gastroenterol Hepatol 2013;9:711-717.
2. Fakhoury M, Negrulj R, Mooranian A, Al-Salami H. Inflammatory bowel disease: clinical aspects and treatments. J Inflamm Res 2014;23:113-120.

3. Leddin D, Tamim H, Levy AR. Decreasing incidence of inflammatory bowel disease in eastern Canada: a population database study. BMC Gastroenterol 2014;14:140.

4. Orel R, Kamhi Trop T. Intestinal microbiota, probiotics and prebiotics in inflammatory bowel disease. World $\mathrm{J}$ Gastroenterol 2014;20:11505-11524.

5. Zhang YZ, Li YY. Inflammatory bowel disease: pathogenesis. World J Gastroenterol 2014;20:91-99.

6. Basso PJ, Fonseca MT, Bonfá G, Alves VB, Sales-Campos H, Nardini V, Cardoso CR. Association among genetic predisposition, gut microbiota, and host immune response in the etiopathogenesis of inflammatory bowel disease. Braz $\mathbf{J}$ Med Biol Res 2014;47:727-737.

7. Freeman HJ. Natural history and long-term clinical course of Crohn's Disease. World J Gastroenterol 2014;20:31-36.

8. Park SJ, Kim WH, Cheon JH. Clinical characteristics and treatment of inflammatory bowel disease: a comparision of Eastern and Western perspectives. World J Gastroenterol 2014;20:11525-11537.

9. Yazisiz V. Similarities and differences between Behçet's disease and Crohn's disease. World J Gastrointest Pathophysiol 2014;5:228-238

10. Chen SJ, Liu XW, Liu JP, Yang XY, Lu FG. Ulcerative colitis as a polymicrobial infection characterized by a sustained broken mucus barrier. World J Gastroenterol 2014;20:9468-9475.

11. Pellicer Z, Santiago JM, Rodriguez A, Alonso V, Antón R, Bosca MM. Management of cutaneous disorders related to inflammatory bowel disease. Ann Gastroenterol 2012;25:21-26.

12. Laranjeira N, Fonseca J, Meira T, Freitas J, Valido S, Leitão J. Oral mucosa lesions and oral symptoms in inflammatory bowel disease patients. Arq Gastroenterol 2015;52:105-110.

13. Trost LB, McDonnell JK. Important cutaneous manifestations of inflammatory bowel disease. Postgrad Med J 2005;81:580-585.

14. Lankarani KB, Sivandzadeh GR, Hassanpour S. Oral manifestation in inflammatory bowel disease: a review. World J Gastroenterol 2013;19:8571-8579.

15. Aguirre A, Nugent CA. Images in Clinical Medicine: Oral Manifestation of Crohn's disease. N Engl J Med. 2015;373:1250

6. Elahi M, Telkabadi M, Samadi V, Vakili H. Association of oral manifestations with ulcerative colitis. Gastroenterol Hepatol Bed Bench 2012;5:155-160.

17. Veloso FT. Extraintestinal manifestations of inflammatory bowel disease: do they influence treatment and outcome? World J Gastroenterol 2011;17:2702-2707.

18. Daley TD, Armstrong JE. Oral manifestations of gastrointestinal diseases. Can J Gastroenterol 2007;21:241-244.

19. Field EA, Allan RB. Review article: oral ulceration-aetiopathogenesis, clinical diagnosis and management in the gastrointestinal clinic. Aliment Pharmacol Ther 2003;18:949-962.

20. Femiano F, Lanza A, Buonaiuto C, Perillo L, Dell'Ermo A, Cirillo N. Pyostomatitis vegetans: a review of the literature. Med Oral Patol Oral Cir Bucal 2009;14:E114E117.

21. Jose FA, Garnett EA, Vittinghoff E, Ferry GD, Winter HS, Baldassano RN, Kirschner BS, Cohen SA, Gold BD, Abramson O, Heyman MB. Development of extraintestinal manifestations in pediatric patients with inflammatory bowel disease. Inflamm Bowel Dis 2009;15:63-68.

22. Danese S, Semeraro S, Papa A, Roberto I, Scaldaferri F, Fedeli G, Gasbarrini G, Gasbarrini A. Extraintestinal manifestations in inflammatory bowel disease. World J Gastroenterol $2005 ; 11: 7227-7236$. 
23. Asquith P, Thompson RA, Cooke WT. Oral manifestations of Crohn's disease. Gut 1975;16:249-254.

24. Lourenço SV, Hussein TP, Bologna SB, Sipahi AM, Nico MM. Oral manifestations of inflammatory bowel disease: a review based on the observation of six cases. J Eur Acad Dermatol Venereol 2010;24:204-207.

\section{Author Affiliations}

Manoela Seadi Pereira* and Maria Cristina Munerato*,

*Faculdade de Odontologia da Universidade Federal do Rio Grande do Sul (UFRGS), Porto Alegre, Brazil

Hospital de Clínicas de Porto Alegre (HCPA), Universidade Federal do Rio Grande do Sul (UFRGS), Porto Alegre,

Brazil 